\title{
Evaluation of Indian Deprived Widow Female Sex Perspective Tests about Pelvis Decline Self-Reported Non-Governmental by-Pass Pharmaceutical Institutions in India
}

ISSN: 2637-8078

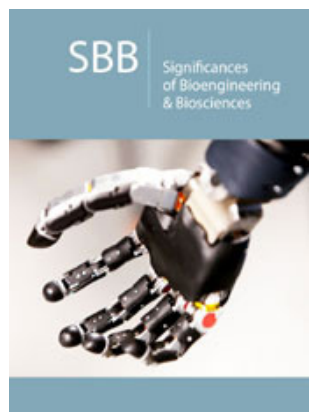

*Corresponding author: Rahul Hajare, Fellow Indian Council of Medical Research, India

Submission: 侮 May 16, 2019

Published: 海 May 21, 2019

Volume 3 - Issue 2

How to cite this article: Rahul Hajare. Evaluation of Indian Deprived Widow Female Sex Perspective Tests about Pelvis Decline Self-Reported Non-Governmental by-Pass Pharmaceutical Institutions in India.Significances Bioeng Biosci.3(2) SBB.000558.2019.

DOI: 10.31031/SBB.2019.03.000558.

Copyright@ Rahul Hajare, This article is distributed under the terms of the Creative Commons Attribution 4.0 International License, which permits unrestricted use and redistribution provided that the original author and source are credited.

\section{Rahul Hajare*}

Fellow Indian Council of Medical Research, India

\section{Abstract}

Female seen poor quality of food living with plastic bikini chair pelvic organ prolapse. Pelvic organ prolapse occurs when the muscles in the pelvis can no longer support its organs, such as the bladder, uterus, or rectum. This can cause one or more of these organs to press down on the vagina. In some cases, this can cause a bulge to form outside of the vagina reduce the pleasure of sexual life.

\section{Introduction}

People who have more sex report higher self-esteem, life satisfaction and quality of life [1]. In contrast, lower frequency of sex and avoiding sex has linked to psychological distress, anxiety, depression and relationship problems, reports by same researcher title: Safe sex: True Principal Health. Sex is, in fact, one of the most basic physiological needs [2]. Yet millions of people spend at least some of their adulthood not having sex. Researcher, from Pune found that up to 19 percent of adults do not engage in sex. This varies by gender and marriage status. The most common reasons for men avoiding sex are erectile dysfunction, chronic medical conditions and lack of opportunity, tough dermatoses head on [3]. Researcher found for example; heart disease patients often avoid sex because they are afraid of a heart attack. Research has shown the same for individuals with cerebrovascular conditions, such as a stroke. Metabolic conditions such as diabetes and obesity reduce sexual activity. In fact, diabetes hastens sexual incline in men by as much as 15 years. Large body mass and poor body image ruins intimacy, which has core to the opportunity for having sex. Research confirms that women more commonly avoid sex than men. In fact, up to 40 percent of women avoid sex some time in their lives. The gender differences start early. More teenage females than teenage males abstain from sex. Women has more likely to avoid sex because of childhood sexual abuse. Pregnant women fear miscarriage or harming the fetus and can also refuse sex because of lack of interest and fatigues. Pain during sex is also a big issue. Chronic pain diminishes the pleasure of the sexual act and directly interferes by limiting positions. The depression and stress it cause can get in the way, as can certain medications for chronic pain. Personality disorders, addiction and substance abuse and poor sleep quality all play major roles in sexual interest and abilities, mouth odour, edentulism is the condition of being reduce the orgasm, lack of enthuse. Many medications, such as antidepressants and anti-anxiety drugs, reduce libido and sexual activity, and, as a result, increase the risk of sexual avoidance. However, few people talk 
with their doctors about their sexual problems. Indeed, at least half of all medical visits do not address sexual issues. Embarrassment, cultural and religious factors, and lack of time may hold some doctors back from asking about the sex lives of their patients. Some doctors feel that addressing sexual issues creates too much closeness to the patient. Others think talking about sexuality can take too much time. Yet while some doctors may be afraid to ask about sex with patients, researcher has shown that patients appear to be willing to provide a response if asked. This means that their sexual problems are not being addressed unless the doctor brings it up. The new ideas presented in this paper not only improved and developed Indian Medicine theory and explored a new theory and method for disease prevention and treatment, but they also integrated traditional medicine with Western medicine to provide better medical services to human beings.

\section{Conclusion}

Elephantiasis has main cause often avoid sex because they have afraid of a risk factors and sexual presentation of extra weight. Heart attack fear. Researcher has shown the same for individuals with must-know rules of casual/ formal sex in so called modern India. Researcher has not recommended his research those females who has diastasis symphysis (vaginal pain from pelvic ligament).

a. Recommendation 1: Researcher has recommended his research for those men women whose Sexual afterglow lasts up to not reach three hours. b. Recommendation 2: Government should earn Loyalty is an important trait has limits.

\section{Executive Summary}

Data has been collected from brutal honesty inservice people attending with fibromyalgia depression close monitoring from pune district pharmaceutical Institutions desperate irreversible loss of fibromyalgia sex lessons learnt from ongoing counselling during bypass of pharmacy institutions. Yet only a few people discuss their sexual problems with their doctors.

\section{Acknowledgment}

I acknowledge for this important work with Honourable Respected Paranjape RS, World Renowned Scientist \& Retired Director \& Scientist 'G High Grade Institute National AIDS Research Institute Pune. This has inspired \& captured the imagination and attention of across the research and pure service.

\section{References}

1. Hajare R (2019) Tuberculosis at the socialized principal pharmaceutical institutions in India without comorbidity in different age groups: An attractive findings. APTI Women's Forum pp. 12-13.

2. Hajare R (2018) Rotational symmetry and corresponding angle of some natural sex restore the appearance of face. MOJ Drug Des Develop Ther 2(5): 210-212.

3. Hajare R (2018) India's adultery law is staggeringly sexist towards women (evaluation without power politics culture in private pharmacy Institutions in India). Pharma Sci Analytical Res J 1(3): 180011. 